\title{
Evaluation of an Energetic Particle Profile Using a Tangential-FIDA Diagnostic in the Large Helical Device*)
}

\author{
Yutaka FUJIWARA $^{1)}$, Shuji KAMIO ${ }^{1)}$, Hiroyuki YAMAGUCHI ${ }^{1)}$, Alvin V. GARCIA ${ }^{3)}$, \\ Luke STAGNER $^{4)}$, Hideo NUGA ${ }^{1)}$, Ryosuke SEKI ${ }^{1,2)}$, Kunihiro OGAWA ${ }^{1,2)}$, Mitsutaka ISOBE ${ }^{1,2)}$, \\ Masayuki YOKOYAMA $^{1,2)}$, Takeo NISHITANI ${ }^{1)}$, William W. HEIDBRINK ${ }^{1,3)}$, \\ Masaki OSAKABE ${ }^{1,2)}$ and LHD Experiment Group ${ }^{1)}$ \\ ${ }^{1)}$ National Institute for Fusion Science, National Institutes of Natural Sciences, 322-6 Oroshi-cho, Toki 509-5292, Japan \\ ${ }^{2)}$ SOKENDAI (The Graduate University for Advanced Studies), 322-6 Oroshi-cho, Toki 509-5292, Japan \\ ${ }^{3)}$ University of California, Irvine, Irvine, CA 92697, USA \\ 4) Oak Ridge Institute for Science and Education, Oak Ridge, TN 37830, USA
}

(Received 10 January 2019 / Accepted 9 May 2019)

\begin{abstract}
A tangential Fast-Ion D Alpha (FIDA) diagnostic is applied to the Large Helical Device (LHD) in order to observe energetic distribution of toroidal circulating energetic particles which are produced by tangential Negative Neutral Beams (NNB). A perpendicular Positive NB (PNB) is used as the diagnostic probe beam of the tangentialFIDA diagnostic in this observation geometry. In order to assess the appropriateness of the tangential-FIDA diagnostic, the experimental result was compared with a Silicon-diode-based Fast Neutral Analyzer (Si-FNA) which was installed on the same line of sight. As a result of the comparison, the tangential-FIDA and the Si-FNA experimental data obtained good linearity in the energy region from $60 \mathrm{keV}$ to $180 \mathrm{keV}$. In addition, an enhanced FIDASIM was applied for analyzing the FIDA on the three-dimensional magnetic configuration fusion device.

(C) 2019 The Japan Society of Plasma Science and Nuclear Fusion Research
\end{abstract}

Keywords: energetic particle, FIDA, FIDASIM, LHD, three-dimensional magnetic configuration

DOI: $10.1585 /$ pfr. 14.3402129

\section{Introduction}

A magnetic confinement fusion reactor requires the combustion maintenance of plasma due to energetic alpha particles produced by fusion reaction. Therefore, it is important to understand the behavior of energetic particles in the magnetic confinement fusion device. To investigate the behavior of energetic particles which were produced by tangentially injected Negative Neutral Beams (NNB) $(<200 \mathrm{keV})$, a Fast-Ion D Alpha (FIDA) diagnostic system was installed on the Large Helical Device (LHD) [1, 2]. In LHD, we have conducted only hydrogen experimental campaigns for several years. The LHD deuterium experimental campaign started in 2017.

In the FIDA diagnostic, the Doppler-shifted $\mathrm{H}$ alpha and $\mathrm{D}$ alpha lights from fast neutrals are utilized as signals of energetic particles, where these fast neutrals are produced by the charge exchange process between fast ions in plasmas and injected NBs [3,4]. The advantages of this charge exchange spectroscopy based fast-ion diagnostic are the locality of the measurement on its line of sight (LOS) and the ease in utilizing the spatial measurements. The interactions between co-going fast ions and Alfvén eigenmode (AE) are reported on the LHD [5]. It is impor-

author's e-mail: fujiwara.yutaka@nifs.ac.jp

*) This article is based on the presentation at the 27th International Toki Conference (ITC27) \& the 13th Asia Pacific Plasma Theory Conference (APPTC2018). tant to evaluate the spatial profile of a fast ion having its kinetic energies largely parallel to the magnetic field lines since the spatial profile of parallel fast-ion component is strongly related to the Alfvénic phenomena. Moreover, it is recognized that the transport of parallel fast ions might suffer more seriously from the influences of turbulences in plasmas than that of perpendicular fast ions.

In this paper, we obtained the data during the hydrogen campaign. On the LHD, we have installed the tangential-FIDA diagnostic for tangential viewing geometry to investigate the confinement property of parallel fast ions. The major challenge of the tangential-FIDA diagnostic on the LHD is the small reaction rate of the charge exchange process for passing fast ions since these fast ions are produced by negative hydrogen-ion based high energy NBs. Another challenge is developing the simulation program of the FIDA diagnostic for the LHD, such as a threedimensional magnetic configuration fusion device.

\section{Measurement Methods}

Figure 1 shows the schematic toroidal cross-section view of the LHD and the geometrical arrangement of the tangential-FIDA diagnostic and the Si-FNA diagnostic on the LHD. The LOSs are horizontally aligned and the radial distributions of Doppler-shifted $\mathrm{H}$ alpha and D alpha emitted from parallel energetic particles can be measured in this 


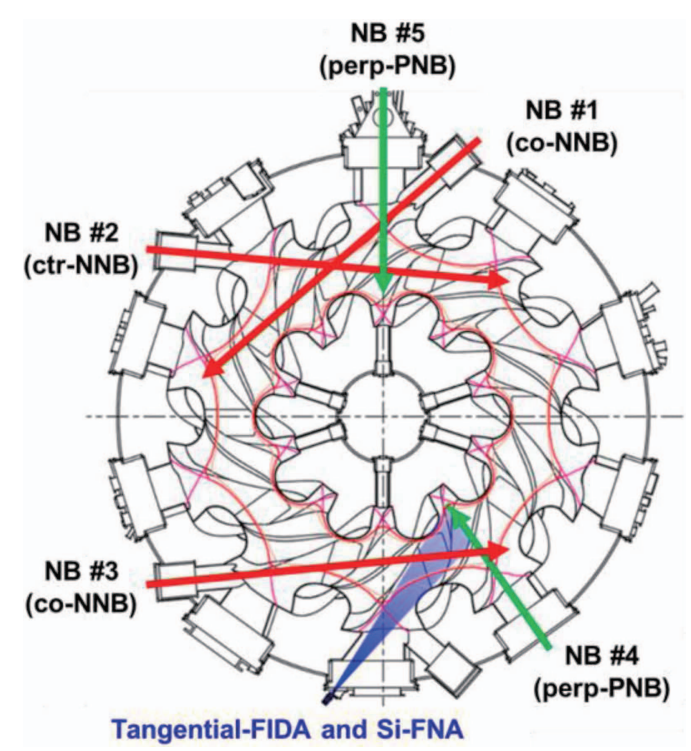

Fig. 1 The schematic toroidal cross-section view of the LHD and the geometrical arrangement of the tangential-FIDA diagnostic and the Si-FNA diagnostic on the LHD.

arrangement. The tangential NB \#1 (co-NNB) and the NB \#2 (counter-NNB) are used as the main sources of energetic particles in this experiment. The other tangential NB \#3 (co-NNB) and the NB \#4 (perp-PNB) can be used as diagnostic probe beams for sources of active neutral particles of the FIDA measurement. However, in this paper, we only use the NB \#4 as the diagnostic probe beam for the measurement of passing energetic particles, because the NB \#3 renders the measurement complicated. The measurement region is located in horizontally elongated poloidal cross section and covers from the magnetic axis to the inboard edge of the LHD plasmas. This corresponds to the major radius $R \sim 2.8 \mathrm{~m}$ to $\sim 3.9 \mathrm{~m}$. The $\mathrm{NB} \# 4$ is required to be modulated for separating the FIDA component from the contribution of the neutral in the peripheral region or the other unnecessary components caused by the injected NB. The Doppler-shifted H alpha and D alpha lights are collected and transferred by the bundled 50 channels optical ?bers which are $400 \mu \mathrm{m}$ in diameter to the spectrometer. The FLP-200 spectrometer is designed and produced by BUNKOKEIKI Co., Ltd. The spectrometer has a grating number of $1200 / \mathrm{mm}$, focal length of $200 \mathrm{~mm}$, and the $F=$ 2.8. A notch filter is placed in the optics to eliminate the cold component of the $\mathrm{H}$ alpha emission. Finally, the collected lights are recorded by the iXon 897 manufactured by ANDOR. The exposure time of the measurement is set to $10 \mu \mathrm{s}$, while the sampling interval is set to $5 \mathrm{~ms}$.

\section{Typical Observation of the Tangential-FIDA}

Figure 2 shows the typical discharge waveforms for the tangential-FIDA measurement. The measurement was performed by the injected NB \#4 $39 \mathrm{keV}$ while the plas-

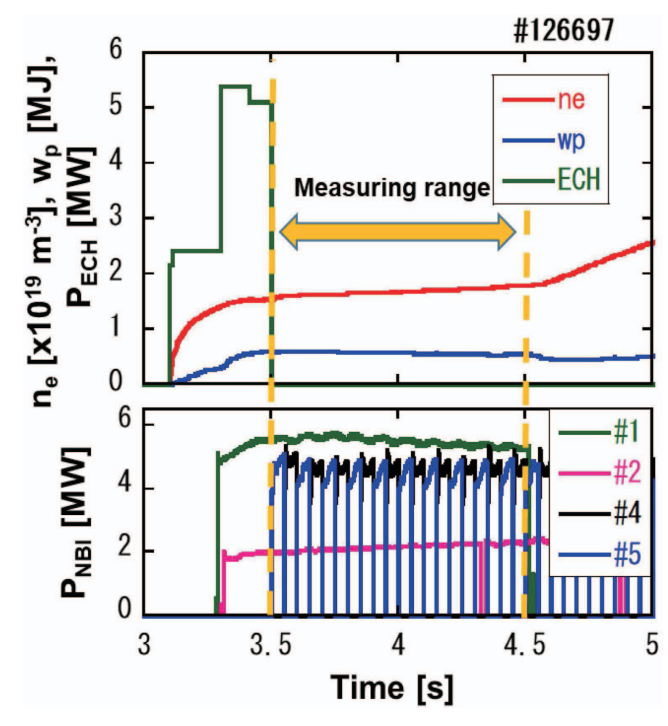

Fig. 2 The waveforms of typical discharge for observation of the FIDA measurement.

mas are sustained by the NB \#1 $185 \mathrm{keV}$ and the NB \#2 $172 \mathrm{keV}$. In this observation geometry, $\mathrm{H}$ alpha light shifts to the red side due to the NB \#1, while $\mathrm{H}$ alpha light shifts to the blue side due to the NB \#2. In this discharge, the typical magnetic configuration in the LHD $(\operatorname{Rax}=3.6 \mathrm{~m}, \gamma=1.254)$ is used and the field strength is set at $2.75 \mathrm{~T}$. The plasma was the pure hydrogen discharge. As mentioned above, the NB \#4 is required to be modulated for separating the tangential-FIDA component. However, because it is not preferable to change the plasma condition during the measurement, the NB \#4 and the NB $\# 5$ are injected in alternation. This means that the NB \#5 is turned off while the NB \#4 is turned on. We must remove the background signal from the tangential-FIDA measurement.

Figure 3 shows the observed tangential-FIDA spectra. In this case, the measured spectral range was set between $643 \mathrm{~nm}$ and $671 \mathrm{~nm}$ on the spectrometer. The intensity of each wavelength was calibrated, including the notch filtered region. The red solid line in Fig. 3 shows the spectra from $t=3.50 \mathrm{~s}$ to $3.55 \mathrm{~s}$ which corresponds to the background measurement with the NB \#5. On the other hand, the blue solid line in Fig. 3 is the spectra from $t=3.55 \mathrm{~s}$ to $3.60 \mathrm{~s}$. These are the spectra with the NB \#4 and they correspond to the foreground measurement. The tangentialFIDA spectra are obtained by subtracting the background spectra from the foreground and are shown by the green solid line in Fig. 3. The several impurity lines are observed both in the foreground and in the background spectra. Fortunately, most of the impurity lines were removed by the background subtraction, as shown in Fig. 3. The bremsstrahlung radiation was also removed by the subtraction because the electron density is almost stationary during the measurement, as shown in Fig. 2. 


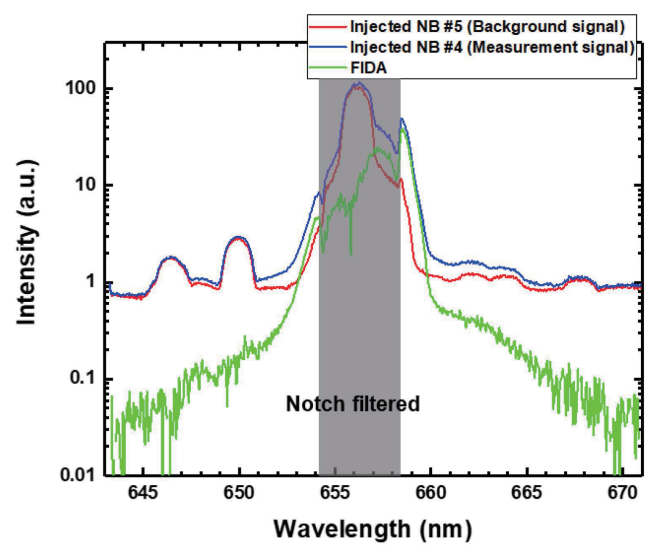

Fig. 3 Measured spectra for $R=3.55 \mathrm{~m}$ when the NB \#4 (perp-PNB) injected (measurement signal) and the NB \#5 (perp-PNB) injected (background signal), and the tangential-FIDA spectra that removed background signal from measurement signal.

\section{Comparison between the Tangential-FIDA and the Si-FNA}

In order to verify the validity of the tangential-FIDA diagnostic, we used the Si-FNA installed on the same measurement line of sight $(R \approx 3.55 \mathrm{~m})$ as the tangential-FIDA [6]. The difference with the tangential-FIDA is that the SiFNA can directly detect energetic particles which are neutralized by the injected NB \#4 and escaped from the magnetic confinement. Also, the two measurements have different weightings in velocity space. The tangential-FIDA measurement integrates over wavelengths that correspond to Doppler shifts produced by energetic particles. For these reasons, the two measurement results are assumed to be in a non-linear relationship.

Figure 4 shows a comparison of the energetic particles signal between the tangential-FIDA results and the Si-FNA results in the experiment. At the FIDA measurement, the FIDA component is produced by the charge exchange reaction $\left(\mathrm{H}_{\text {fast }}^{+}+\mathrm{H}_{\mathrm{NB}} \rightarrow \mathrm{H}_{\text {fast }}(n=3)+\mathrm{H}_{\mathrm{NB}}^{+}\right)$. On the other hand, Si-FNA measurement is produced by the charge exchange reaction $\left(\mathrm{H}_{\text {fast }}^{+}+\mathrm{H}_{\mathrm{NB}} \rightarrow \mathrm{H}_{\text {fast }}\right.$ (sum of $n=1,2$, $3 \ldots)+\mathrm{H}_{\mathrm{NB}}^{+}$). These are not the exact same reactions. However, the ratio between the two cross sections is almost constant in the energy range of $50 \sim 200 \mathrm{keV} / \mathrm{amu}$, as shown in the ADAS database [7]. Therefore, the energy dependence on the ratio of the cross section between the two reactions is negligible. In Fig. 4, the vertical axis is the emission intensity (a.u.) of the tangential-FIDA and the horizontal axis is the count number of the Si-FNA. Measurement was performed in the measuring range shown in Fig. 2. The plots in Fig. 4 are six points corresponding to $60 \sim 80 \mathrm{keV}, 80 \sim 100 \mathrm{keV}, 100 \sim 120 \mathrm{keV}, 120 \sim 140 \mathrm{keV}$, $140 \sim 160 \mathrm{keV}$, and $160 \sim 180 \mathrm{keV}$, respectively. As shown in the figure, the two measurement signals show good linearity. This seems to indicate the validity of the tangentialFIDA diagnostic.

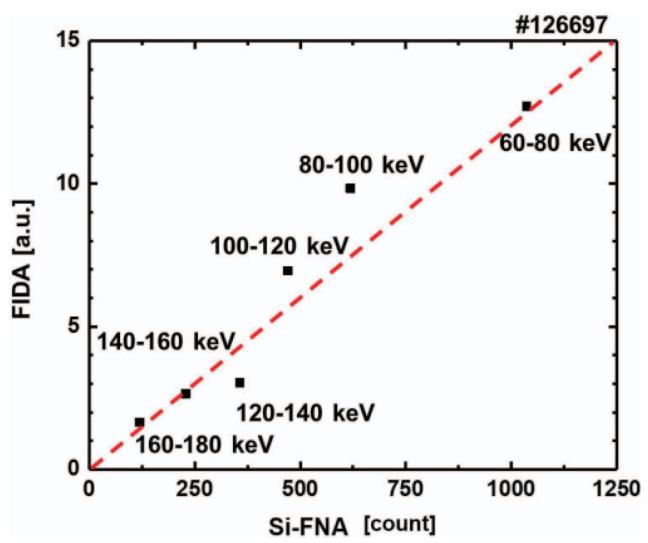

Fig. 4 Comparison of the energetic particles signal between the tangential-FIDA results and the Si-FNA results in the experiment.

\section{FIDASIM Developed for the LHD}

The FIDASIM is a famous FIDA analysis tool [8]. However, it was not possible to use the FIDASIM in a three-dimensional magnetic configuration fusion device, such as a helical type fusion device. This is because the FIDASIM is a simulation code developed based on an axisymmetric configuration, which is a two-dimensional magnetic configuration fusion device such as a tokamak type fusion device [9]. Recently, the FIDASIM enhanced to calculate in three-dimensional geometry. The FIDASIM requires the distribution function, plasma profiles, magnetic equilibrium, and diagnostic geometry to be in a specific format. A PREFIDA is IDL code that takes the required inputs, checks their validity, and transforms them into a form the FIDASIM understands. In this work, a code was developed that uses the PREFIDA to produce the inputs files needed to run the FIDASIM in three-dimensional geometry. The most important analysis in the FIDA diagnostic is distinguishing the FIDA signal from other bright sources of light in the same region. For such occasions, the FIDASIM is able to calculate the light of various sources and expect spectra profiles of the FIDA measurement for each LOS by inputted plasma parameter, magnetic configuration, diagnostic beam information, LOS geometry, and phase-space distribution of energetic particles.

Figure 5 shows the schematic 3D mesh view of the calculation region for the FIDASIM on the LHD and the magnetic configuration view on the typical experimental case. For the developed code, the input data must be based on the Cartesian coordinates. We located the origin of the Cartesian coordinates system, and $x$ axis was an opposite direction of the perp-NB \#4, $y$ axis was perpendicular direction to $x$ axis on the equational plain, and $z$ axis was in the vertical direction. In the case of Fig. 5, we determined the calculation region to be from $2.7 \mathrm{~m}$ to $4.7 \mathrm{~m}$ on $x$ axis, from $-0.5 \mathrm{~m}$ to $0.5 \mathrm{~m}$ on $y$ axis, and from $-0.6 \mathrm{~m}$ to $0.6 \mathrm{~m}$ on $z$ axis. Also, we made 40 grids for $x$ axis, 21 grids for $y$ axis, and 25 grids for $z$ axis with $5 \mathrm{~cm}$ increments in 


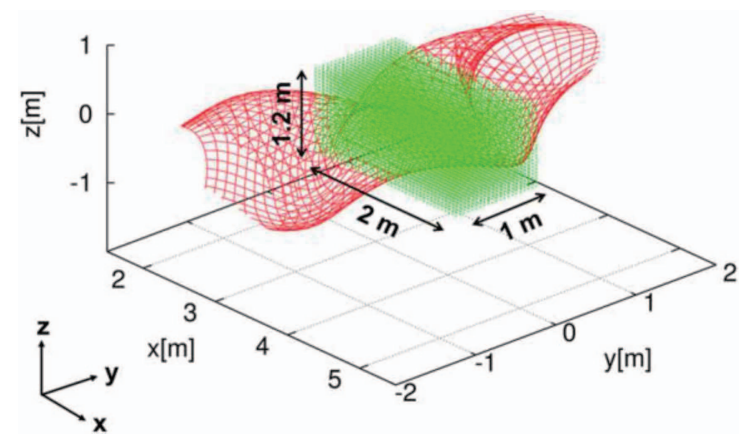

Fig. 5 The schematic 3D mesh view of the calculation region for the FIDASIM on the LHD and the magnetic configuration view on the typical experimental case.

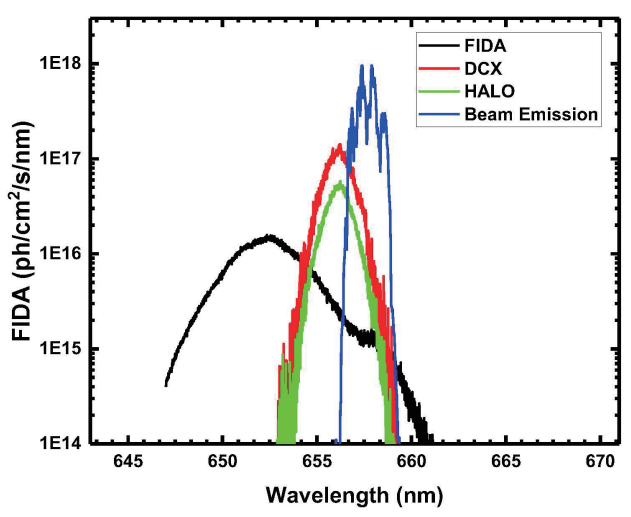

Fig. 6 Various sources of light in the $\mathrm{H}$ alpha spectral band were calculated by the FIDASIM on the typical experimental case.

between. In this paper, we calculated the phase-space distribution of energetic particles on the calculating region by the GNET for the typical experimental case. The GNET considers the equilibrium, and uses plasma and beam parameters to calculate the phase-space distribution of energetic particles.

Figure 6 shows various sources of light in the $\mathrm{H}$ alpha spectral band calculated by the FIDASIM in the typical experimental case. The black solid line is the signal FIDA component. The red solid line is the direct charge exchange (DCX) component signal which comes from the charge exchange reaction between beam neutrals and thermal ions. The green solid line is the HALO signal which comes from the newly created thermal neutrals which travel ballistically and charge exchange with other thermal ions creating more neutrals after neutralization. The blue solid line is the beam emission signal. This FIDASIM analysis result requires attention because the calculation of FIDASIM in Fig. 6 considers only the phasespace distribution of energetic particles from NB \#2 as input data, thus only the blue shift FIDA component is shown. To be mentioned, the FIDA measurement result contained energetic particles injected from NB \#1 and NB
\#2, as shown in Fig. 2.

As a result of comparison with the FIDA experimental data and the FIDASIM analysis results, the experimental spectra around $659 \mathrm{~nm}$ seems to be dominated by the beam emission component. In the measured spectra between $655 \mathrm{~nm}$ and $658 \mathrm{~nm}$ shown in Fig. 3, there are two peaks that may refer to the DCX component and the beam emission component, respectively. Regarding these points, it seems that the FIDA experimental data and the FIDASIM analysis results are consistent. However, in the FIDA component, the analysis results are not consistent. The FIDA component of FIDASIM is about ten times larger than the FIDA experimental result. This analysis data of the FIDASIM is an initial calculation result. In order to verify with experimental results, it is necessary to optimize the conditions necessary for calculating the FIDASIM. For example, we must set the area to be calculated, set the size of the mesh, and set the number of particles to be used for calculations. Also, we must consider the validity of the input phase-space distribution. We will conduct further research to make the FIDASIM analysis succeed.

\section{Conclusion}

In order to measure the tangential-FIDA diagnostic, we experimented with patterns that alternately injected the NB \#4 (perp-PNB) and the NB \#5 (perp-PNB). We succeeded in removing the background spectra from the measurement spectra to obtain the tangential-FIDA spectra. In order to assess the appropriateness of the tangentialFIDA diagnostic, the experimental result was compared with the Si-FNA which was installed on the same line of sight. As a result of the comparison, the energetic particle flux calculated by the tangential-FIDA was consistent with the measurement with the Si-FNA as shown in Fig. 4. The enhanced FIDASIM was applied for analyzing the FIDA diagnostic on the three-dimensional magnetic configuration fusion device. The initial calculation result of the FIDASIM for the LHD was successfully obtained as shown in Fig. 6. We inputted data of the phasespace distribution of energetic particles, which was calculated by the GNET. The energy distribution was consistent with the Si-FNA. The two peaks in the wavelength range $655 \sim 658 \mathrm{~nm}$ shown in Fig. 3 can be reproduced by the FIDASIM, which means the emissions from the bulk plasma collisions (DCX, HALO, Beam Emission) in the FIDASIM seem to be consistent with the FIDA measurement results. However, the FIDASIM analysis results are not in good agreement with the FIDA experimental data. We will study further in order to correlate between the tangential-FIDA spectra and the FIDASIM calculation results.

\section{Acknowledgments}

This research was supported by the NINS program of Promoting Research by Networking among Institu- 
tions (Grant Number 01411702), the NIFS International Collaboration Research programs (NIFS18/KLPR047 and NIFS07/KLPH004), and the LHD project budget (ULRR006, ULRR035, ULRR036, and ULRR702). A part of this work was performed on "Plasma Simulator" (FUJITSU FX100) of NIFS with the support and under the auspices of the NIFS Collaboration Research program NIFS18KNST135.

[1] M. Osakabe et al., Rev. Sci. Instrum. 79, 10E519 (2008).
[2] T. Ito et al., Plasma Fusion Res. 5, S2099 (2010).

[3] W.W. Heidbrink et al., Plasma Phys. Control. Fusion 46, 1855 (2004).

[4] Y. Luo et al., Rev. Sci. Instrum. 78, 033505 (2007).

[5] M. Osakabe et al., Nucl. Fusion 46, S911 (2006).

[6] M. Osakabe et al., Rev. Sci. Instrum. 72, 788 (2001).

[7] H.P. Summers, The ADAS User Manual, version 2.6 (2004). http://www.adas.ac.uk

[8] W.W. Heidbrink et al., Commun. Comput. Phys. 10, 716 (2011).

[9] W.W. Heidbrink, Rev. Sci. Instrum. 81, 10D727 (2010). 